\title{
Pulmonary Sequestration With Fungus Infection Presenting as Highly Elevated Carcinoembryonic Antigen
}

Wei luo

People's Hospital of Leshan

Tong-chen Hu

People's Hospital of Leshan

lincheng luo

People's Hospital of Leshan

Ya-lun Li (D 59968642@qq.com )

Sichuan University West China Hospital

\section{Case report}

Keywords: pulmonary sequestration, Aspergillus infection, carcinoembryonic antigen

Posted Date: April 7th, 2021

DOl: https://doi.org/10.21203/rs.3.rs-370411/v1

License: (c) (i) This work is licensed under a Creative Commons Attribution 4.0 International License.

Read Full License 


\section{Abstract}

Background: Pulmonary sequestration (PS) associated with massive hemoptysis, hemothorax, and elevated tumor markers or even diagnosed with lung malignancy has been reported many times. But all these clinical features have never been reported in the patient diagnosed with PS without malignant disease.

Case presentation: A 45-year-old man with PS presented with massive hemoptysis, hemothorax, extremely elevated carcinoembryonic antigen (CEA) in the pleural effusion, was initially misdiagnosed with advanced lung carcinoma, and was finally diagnosed with PS with Aspergillus infection.

Conclusions: PS is rarely concurrent with lung cancer; most of the time, it is easily misdiagnosed as a malignancy, especially when presenting with a fungal infection, which could remarkably elevate CEA in the pleural effusion.

\section{Introduction}

Pulmonary sequestration (PS) is a congenital pulmonary malformation with an estimated incidence of $2.2-6.6 \%$. It can be defined as a part of the lung that has no normal communication with the bronchial tree and receives its blood supply instead from the systemic arteries (1). In recent decades, there have been several reports of PS associated with massive hemoptysis, hemothorax, and elevated tumor markers such as carcinoembryonic antigen (CEA) or even diagnosed with lung malignancy (2-4). However, these clinical features mentioned above are rarely seen in the patient diagnosed with PS without malignant disease. Here, we describe a patient with all three presentations who was nearly diagnosed with primary lung malignancy but was finally diagnosed with PS with Aspergillus infection.

\section{Case Presentation}

A 45-year-old man presented with massive hemoptysis for 3 days and breathlessness on exertion with a history of 30 pack-years of smoking. He had moderate fever but no night sweats, anorexia or weight loss. The patient had a respiratory rate of 28 breaths/min and oxygen saturation of $91 \%$ on room air. Physical examination revealed percussive dullness and diminished breath sounds on the left side of the chest. He had moderate leukocytosis $(16,500 / \mu \mathrm{L})$ and procalcitonin $(11.3 \mathrm{mg} / \mathrm{L})$, and serum CEA was normal $(2.2$ $\mathrm{ng} / \mathrm{mL}$ ). Other laboratory tests were unremarkable. Enhanced chest computed tomography (CT) scans revealed a cystic mass that was mildly enhanced in the left lower lobe supplied by an anomalous artery arising from the descending thoracic aorta with massive pleural effusion (Fig. A). Therefore, he was initially diagnosed with PS with inflammatory pleural effusion. Nevertheless, tube thoracostomy revealed hemothorax, while the CEA in the pleural effusion reached up to $98 \mathrm{ng} / \mathrm{ml}$, and cytological analysis demonstrated that neutrophils were the major type of leukocyte. These findings complicated the diagnosis such that we could not exclude the possibility of lung cancer, while lobectomy was not appropriate in the patient's condition. The hemoptysis was stopped after bronchial arterial embolism 
(BAE) with coils and polyvinyl alcohol (Fig. B, C), and bronchoscopy and thoracoscopy were performed. Bronchoscopy was unremarkable, and thoracoscopy showed some lesions on the parietal pleura, bloody effusion and fibrous adhesions (Fig. D). However, the histopathology of the pleural lesion only implied chronic inflammation with some necrosis. CT-guided needle biopsy of the retrocardiac mass was performed twice (Fig. E). None of the histopathological and cytological examinations suggested malignancy, and only elevated neutrophils and Aspergillus hyphae emerged. Because there was no evidence of malignancy, left lower lobectomy was performed, and the lesion was removed thoroughly (Fig. F). Postsurgical histopathology examination confirmed the diagnosis of intralobar pulmonary sequestrations (IPSs) and the existence of septate hyphae as well as some fibrinoid necrosis (Fig. G, H). After the surgery, the CEA in the pleural effusion and the temperature returned to normal. The patient recovered very well and was discharged 7 days later.

\section{Discussion}

We describe a rare case of PS with Aspergillus infection that presented as massive hemoptysis, hemothorax, extremely elevated CEA in the pleural effusion and initial radiological findings mimicking advanced lung carcinoma, complicating the therapeutic strategy (no surgery was performed first) and subjecting the patient to repeated biopsies, unnecessary anxiety and high medical costs. Thus far, at least seven cases of lung cancer with PS have been reported (5). However, up to $21 \%$ of PS cases have been misdiagnosed as lung cancer among 2,625 cases over a 10-year period in China (6).

An oncologic correlation worth mentioning is the relationship between the hemothorax and the remarkably elevated CEA in the pleural effusion in this case. Given the sensitivity and specificity of CEA in diagnosing malignancy, the patient was strongly suspected of having advanced lung cancer at the beginning, and we were only able to rule out this suspicion after surgery. There were some reports of PS patients with elevated serum CEA and carbohydrate antigens 19 - 9 and 125 (CA199, CA125), but the corresponding titer were very low (less than $10 \mathrm{ng} / \mathrm{mL})(4,7,8)$. Noguchi et al. and Wei Tang separately found elevated levels of CEA in some patients with allergic bronchopulmonary aspergillosis $(9,10)$. These findings suggest that CEA is correlated with the inflammatory activity of the fungus, while the underlying mechanism remains unclear. In this case, we hypothesized that the Aspergillus infection caused the elevation in CEA in the pleural effusion because after removing the lesion, the level of CEA quickly returned to normal.

\section{Conclusions}

PS could be associated with elevated tumor markers, especially lung Aspergillus infection, which could result in a remarkably increased CEA in either the pleural effusion or serum, leading to an easy misdiagnosis of advanced lung malignancy.

\section{Declarations}


Approval was obtained from our Institutional Review Board and patient for this case report.

All the authors are qualified for the authorship and agree to submit this paper.

All data and materials are available.

All authors declare no conflict of interest.

There is no funding support for this case.

Doc Wei Luo, lincheng Luo and Yalun Li worked together for the treatment of this patient and prepared for the manuscript. Doc Tongcheng Hu did the surgery.

Acknowledgements: Not applicable.

\section{References}

1. Corbett HJ, Humphrey GM. Pulmonary sequestration. Paediatr Respir Rev. 2004;5(1):59-68.

2. Kleffner T, Holzer M, Hulskamp G, Feindt P, Groetzner J. Acute hemoptysis and pulmonary hemorrhage after judo as presentation of intralobar sequestration. Thorac Cardiovasc Surg. 2013;61(2):172-4.

3. Di Crescenzo V, Laperuta P, Napolitano F, Carlomagno C, Garzi A, Vitale M. Pulmonary sequestration presented as massive left hemothorax and associated with primary lung sarcoma. BMC Surg. 2013;13(Suppl 2):34.

4. Teng G, Nie X, Wang D. Association of pulmonary sequestration with elevated serum cancer antigen 125 levels: a case report. J Int Med Res. 2020;48(2):300060520903871.

5. Okamoto T, Masuya D, Nakashima T, Ishikawa S, Yamamoto Y, Huang CL, et al. Successful treatment for lung cancer associated with pulmonary sequestration. Ann Thorac Surg. 2005;80(6):2344-6.

6. Wei Y, Li F. Pulmonary sequestration: a retrospective analysis of 2625 cases in China. Eur $\mathrm{J}$ Cardiothorac Surg. 2011;40(1):e39-42.

7. Lin WC, Tseng YT, Chang YL, Lee YC. Pulmonary tumour with high carcinoembryonic antigen titre caused by chronic propolis aspiration. Eur Respir J. 2007;30(6):1227-30.

8. Ahn YH, Song MJ, Park SH. Intralobar Pulmonary Sequestration Showing Increased Serum CA19-9. Tuberc Respir Dis (Seoul). 2012;72(6):507-10.

9. Tang W, Deng S, Luo L, Luo B, Hu C, Niu R, et al. Allergic Bronchopulmonary Aspergillosis With Elevated CEA Is Infrequent. Arch Bronconeumol. 2020;56(4):256-7.

10. Noguchi T, Yamamoto K, Moriyama G, Saito Y, Kyoyama H, Mikami S, et al. Evaluation of serum levels of carcinoembryonic antigen in allergic bronchopulmonary aspergillosis. J Nippon Med Sch. 2013;80(6):404-9. 


\section{Figures}

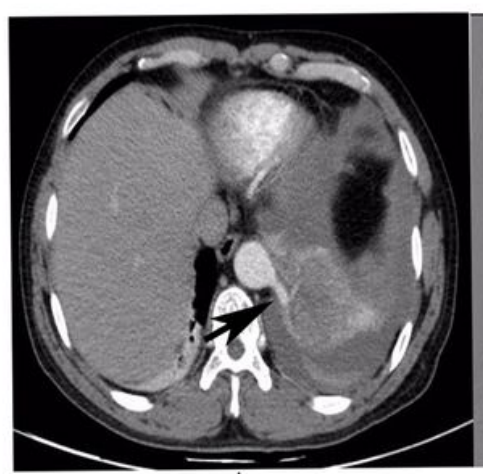

A

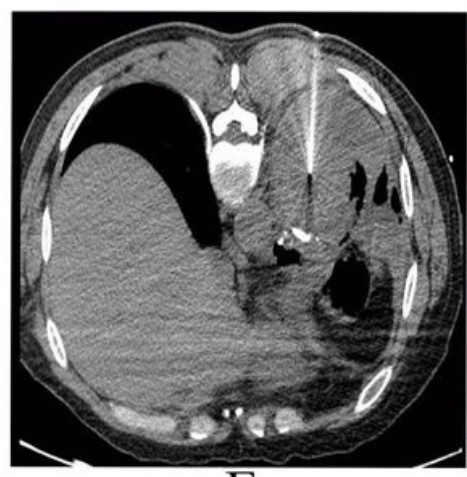

$\mathrm{E}$

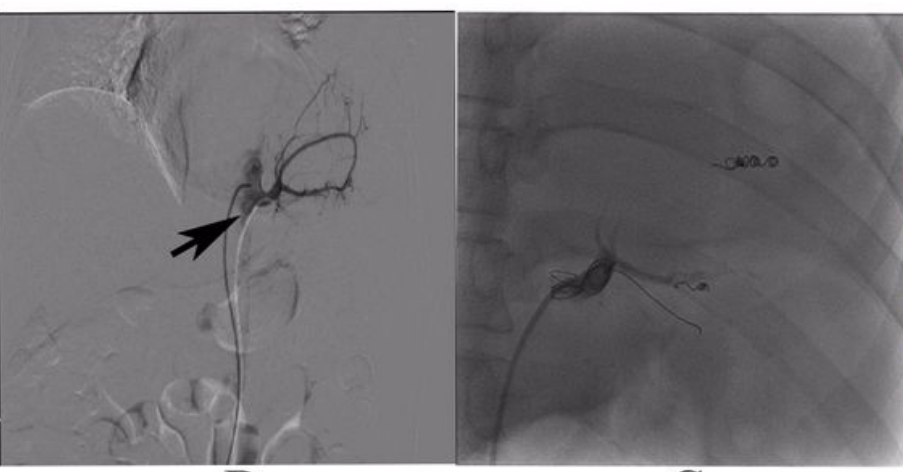

$\mathrm{B}$

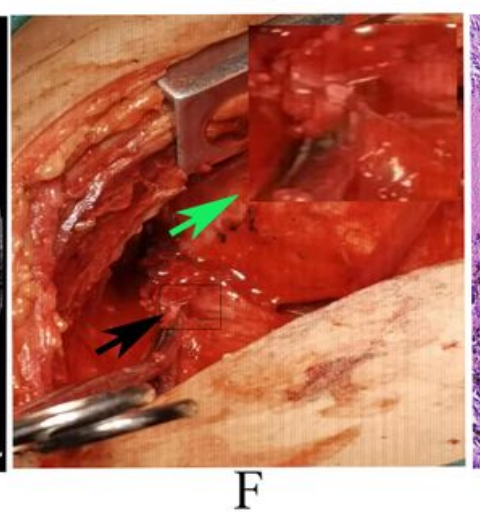

$\mathrm{C}$

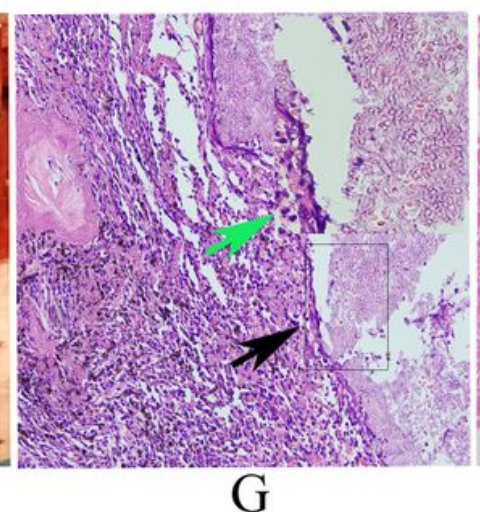

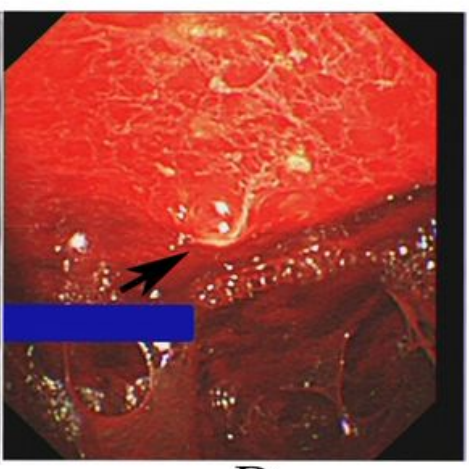

$\mathrm{D}$

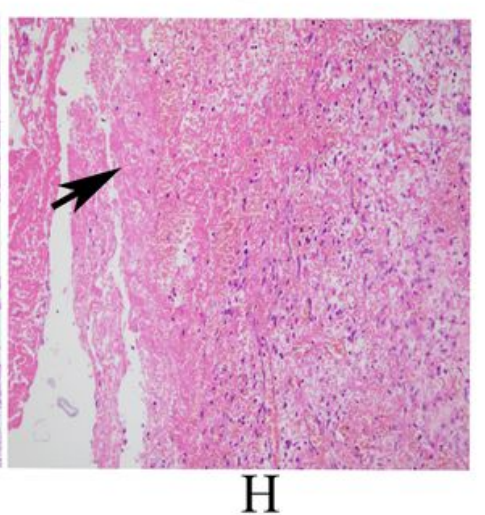

\section{Figure 1}

Figure A. Axial section of thoracic contrast-enhanced CT showing a heterogeneous mass at the posterior basal segment of the left lower lobes with a massive pleural effusion (black arrow). Figure B. Bronchial artery angiography showing an aberrant artery arising from the descending thoracic aorta (black arrow). Figure C. The aberrant artery embolized with coils and polyvinyl alcohol. Figure D. Thoracoscopy showing a lesion on the parietal pleura, bloody effusion and fibrous adhesions (black arrow). Figure E. CT-guided needle biopsy showing the needle in the mass. Figure F. Lobectomy showing the aberrant artery as well as the coils (black arrow and rectangle); the right upper corner shows an enlarged view of the artery and coil (green arrow). Figure G. Septate hyphae in the resected mass with a chronic inflammatory reaction (black arrow and rectangle); the right upper corner shows an enlarged view of the septate hyphae (green arrow). H\&E staining, original magnification $\times 200$. Figure $\mathrm{H}$. Lobectomy showing fibrinoid necrosis with inflammatory cell infiltration. H\&E staining, original magnification $\times 200$. 